\title{
Tideland Animal AR: Superimposing 3D Animal Models to User Defined Targets for Augmented Reality Game
}

\author{
Youngo Lee and Jongmyong Choi \\ Computer Engineering, Mokpo National University \\ \{youngho,jmchoi\}@mokpo.ac.kr
}

\begin{abstract}
With the development of computer vision, mobile platform, and information technologies, there have been many mobile augmented reality applictutions, contents $>$ and services. The technology of augmented reality has been developed, to many application areas such as books, games, advertisements, and tour guide systems. However, most vision-based augmented reality applications have to train some physical objects patterns or paper markers before using it. If the physical objects for the applications are destroyed or damaged, users have to buy new objects or print them out agdin. To reduce the inconvenience, there has been developed technology that users take picturgs of $2 D$ makers what users want and superimpose content on it in real-time. In this paper, we present an application that superimposes $3 D$ animal models living in tideland sequentially whenever users make image targets in real time. We implemented 3D models of 10 animals in iddeland, and a mobile application to display the animals on smart phone.
\end{abstract}

Keywords: augmented reality, userddefined target, tideland, 3D animal model

\section{Introduction}

With the developmen of mobile augmented reality (AR) enabling technology, there have been many mobile AR applications, contents, and services [1-2]. The technology of augmented reality has been developed to many application areas such as books, games, advertisements, and tour guide systems. These applications show 3D animated content or animated graphics on a physical object through various types of displays such as monitor screen, HMD, or smart phones [3- 5].

However, most vision based augmented reality applications have to train physical objects patterns or paper markers before we use it [6]. Thus, if the physical objects for the AR application has destroyed or damaged, users have to buy new object or print it out again. To reduce inconvenience, there has been developed technology that users take pictures of 2D makers what users want and superimpose content on it in real-time. Qualcomm provides developers with Vuforia SDK which has many interesting features including user defined image targets.

In this paper, we present an application that superimposes 3D animal models living in tideland sequentially whenever users make image targets in real time. To prove the effectiveness of this technique, we implemented a mobile augmented reality application for smart phone with 3D animals in tideland. We select 10 animals living in tideland and make 3D animated models of them. With the vuforia SDK, we implement an application for smart phone which shows 3D tidal animals on 2D plane where users take 
a picture through a smart phone camera. We make a queue of dataset for tracking $2 \mathrm{D}$ plane taken by users so that when users take a picture of 2D plane, it shows tidal animal sequentially.

This paper consists of five sections. In Section 2, we will summarize background. After then, we describe implementation of AR eBook for an eBook in Section 3. Finally, we will reveal our conclusions in Section 4.

\section{Background}

\subsection{Augmented Reality}

Augmented reality (AR) applications are classified into several groups according to the type of input sensor for tracking. There are vision-based AR, outdoor- or GPS-based AR, hybrid AR and others. Vision-based augmented reality tecknology is based on computer vision algorithm to process feature points of $2 \mathrm{D}$ planes or $3 \mathrm{D}$ polyhedrons from camera images in real world and superimpose 3D content on them in real îme. ARToolkit, ARTag, vuforia, etc are representative toolkits for vision based AR. In outdoor, it is difficult to extract feature points for tracking. Outdoor (GPS-based) augmented reality technology shows geo-information nearby user's current position. It extracts user's global position on earth from a GPS sensor. Different with viewing $2 D$ map shown in smart phone, it shows geoinformation according to selected direction by users.

There are several platforms and SDKs torealize vision-based augmented reality. ARtoolkit and ARtag are $\mathrm{C} / \mathrm{C}++$ open søuree platforms which support tracking 2D marker and displaying openGL [8]. BazAR is a computer vision library based on feature points detection and matching [9]. In particular, it is able to quickly detect and register known planar objects in images. The Point Cloud Library (PCL) is a standalone, large scale, open project for 2D/3D image and point cloud processing [10]. Qualcomm Vuforia is a software platform for Android and iOS that enables app to see images from a Vuforia target database on the device or in the cloud [7]. It allows users make miobile AR apps which track objects such as tow-dimensional planar images, three-dimensional cube, and cylinder-shape object. It also supports real time text recognition and user defind targets class. So, users can make dataset for image tracking by taking pictures that users want in real time.

Qualcomm provides Yuforia sdk for android, IOS and package for unity3d [7]. The userDefinedTargetBuildingBehaviour class is a class which generates dataset from photos taken by a user in real time. This class provides a developer with functions to generate dataset from photo ancto scan automatically. By using this class and others in vuforia SDK, we implemented our application which makes dataset whenever a user take pictures and displays 3D models on it.

\subsection{Tideland and Animals}

Tidelands are territory between the high and low water tide line of see coasts, and lands lying under the sea beyond the low water limit of the tide. In general, tidelands are important area for wild animals such as a mudskipper, a sand crab, a sea shell, and various kinds of animals. In past, tidelands were considered as non-useful land, however, today they are considered as useful land because of its economic values. Figure 1 shows a mudskipper and a sand crab in tideland. 


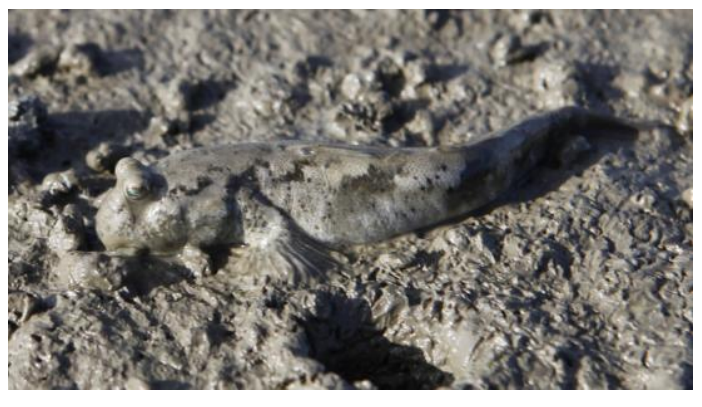

(a) a mudskipper

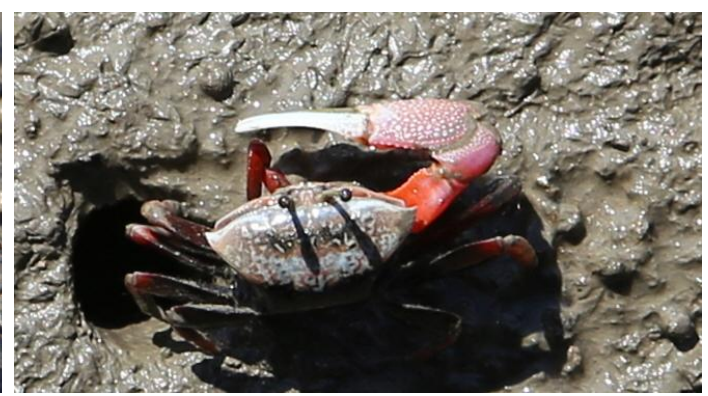

(b) a sand crab

Figure 1. Animals in tideland. (a) is a mudskipper and (b) is a sand crab

\section{Tideland Animal AR}

Our proposed AR application named 'Tideland Animar AR' is designed for children who are going to study tidelands and its animals and to visit tidelands. Alter reading text book about tideland, children may play with this mobile application in their home, so that they watch animals in tideland before going to there. The childrep take a picture of a plane such as photo frames, books, and posters in house with their smart phone camera, and then the smart phone shows a 3D animal model over the plane throughthe video camera.

We gathered text books and information about tideland and its animals, and visited tideland in South Korea to take pictures and videos of them. We took pictures and videos about 50 kinds of animals. With these materials, we select 10 funny and important animals for 3D modeling. For the mobile platform, the models have small number of polygons and high quality textures.

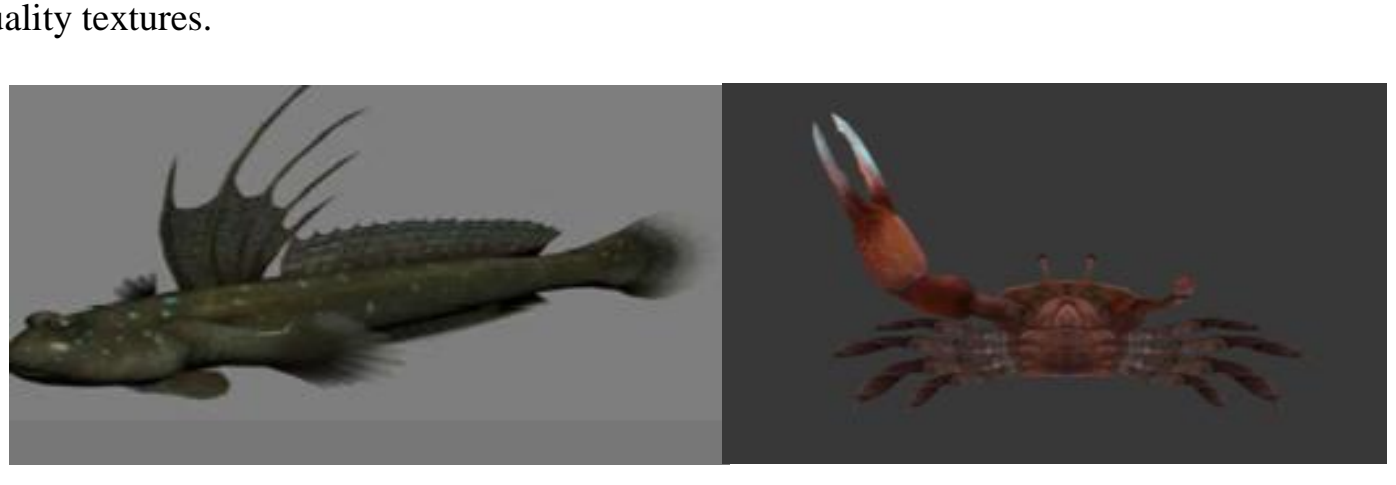

(a) 3D model of a mudskipper

(b) 3D model of a sand crab

\section{Figure 2. 3D animal models}

The vuforia SDK provides functions to make augmented reality applications. With vuforia SDK, we augment 3D models on 2D plane, cylinder shape, and cubical shape. It also provides functions to make user defined image target. However, to superimpose several 3D models, we need to develop a data structure for managing several 3D models and image targets.

First, we make dataset array and pause it for a while. Before adding a new dataset, check whether the array full or not. If the array is full, then remove the last one so that we can add new one. Then we assign a 3D model to the new dataset. Whenever a user takes a picture, we save the new data into array and assign its corresponding 3D model sequentially, and remove 
the last one repeatedly. By this procedure, several 3D models appear repeatedly whenever a user takes photos. Figure 3 shows the relationship between dataset and connected 3D models.

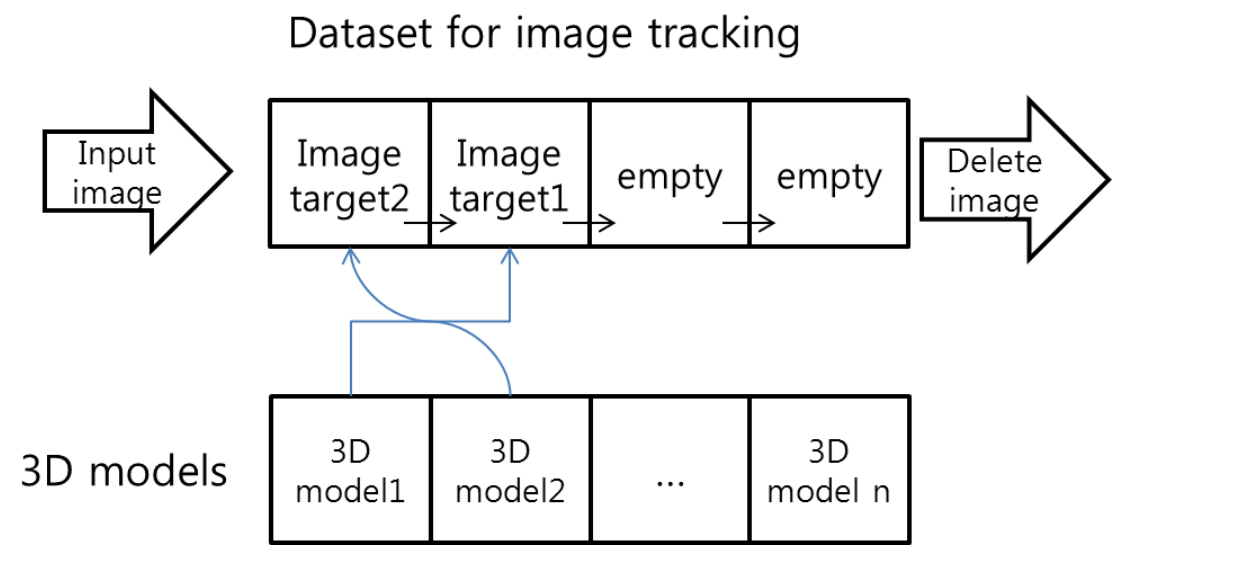

Figure 3. Dataset is a queue and each array connects with 3D model sequentially

\section{Implementation}

We used unity 3D and vuforia SDK for implementation. We use ten 3D animal models in tideland as shown in Figute 4. Figure 4 (a) and (c) are images before taking pictures and Figure 4(b) and (d) shows 3D models of a shrimp and a mudskipper on text books.

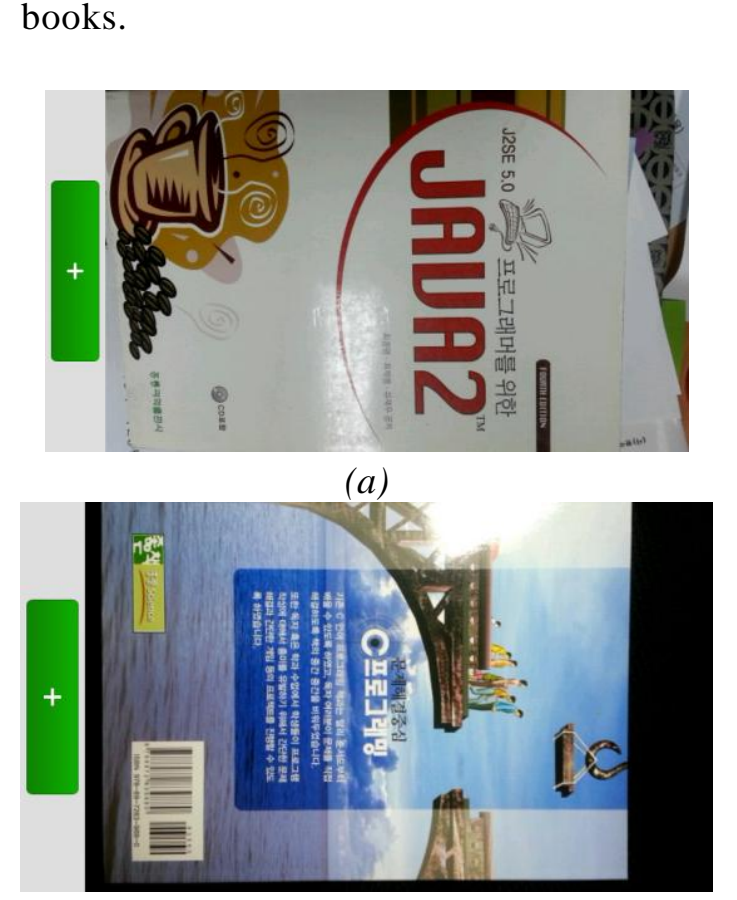

(c)

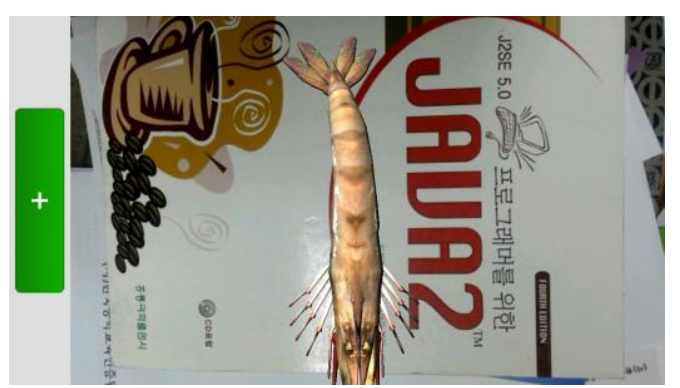

(b)

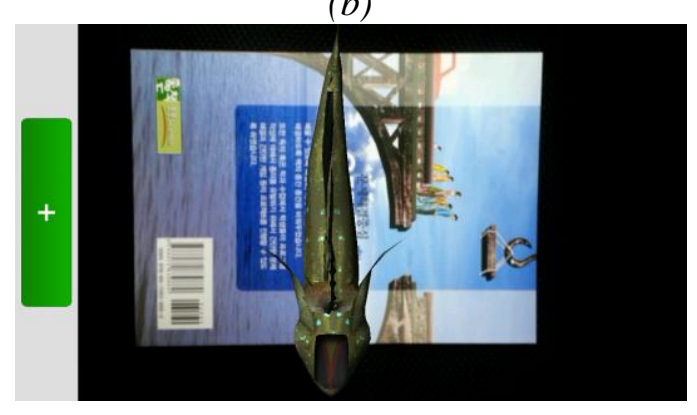

(d)

Figure 4. Examples. (a) and (c) are photos taken by a user. (b) and (d) show 3D models on the photos 


\section{Conclusion and Future works}

In this paper, we present an application that superimposes 3D animal models living in tideland sequentially whenever users make image targets in real time. With our application, users do not need to re-make or buy markers for AR tracking, and they can superimpose 3D content in any $2 \mathrm{D}$ plane in house or in office. With the proposed method, when a user take photos directly, the mobile application process the photos and shows 3D animal models on them in real time. Users can put 3D animal models any place they want to. In future, we have planned to evaluate user study so that people can enjoy our augmented reality applications.

\section{References}

[1] P. Milgram, H. Takemura, A. Utsumi, F. Kishino, "Augmented Reality: A Class of Displays on the RealityVirtuality Continuum", Proceedings of the SPIE Conference on Telemanipulator and Telepresence Technologies, vol. 2351, (1995), pp. 282-292.

[2] R. Azuma, Y. Baillot, R. Behringer, S. Feiner, S. Julier and B. MàcIntyre, "Recent advances in augmented reality, Computer Graphics and Applications", IEEE , vol. 21, no. 6, (2001) November/December, pp. 34-47.

[3] T. Ha, Y. Lee and W. Woo, Digilog book for temple bell tolling experience based on interactive augmented reality", Virtual Reality, vol. 15, no. 4, (2010), pp. 295-309.

[4] M. Billinghurst, H. Kato and I. Poupyrev, "The MagicBook ${ }^{\bullet}$ moying seamlessly between reality and virtuality", Computer Graphics and Applications, IEEE, vol. 21, no. 3, (2001) May/June, pp. 6-8.

[5] N. Taketa, K. Hayashi, H. Kato and S. Noshida, "Virtual Pop-Cp Book Based on Augmented Reality, Human Interface and the Management of Information", Interasting in Information Environments, Lecture Notes in Computer Science, vol. 4558, (2007) pp 475-484.

[6] D. Beier, R. Billert, B. Bruderlin, D. Stichling and B. Kleinjohann, "Marker-less vision based tracking for mobile augmented reality", The Second IEEE and ACM International Symposium on Mixed and Augmented Reality, (2003), pp. $258-259$

[7] Vuforia, http://www.vuforiacom

[8] D. F. Abawi, J. Bienwåld and R. Dorner. "Accuracy in Optical Tracking with Fiducial Markers: An Accuracy Function for ARToolKit', In Proceedings of the 3rd IEEE/ACM International Symposium on Mixed and Augmented Reality (ISMAR '04), (2004).

[9] bazar. http://c vlab.epfl.ch/software/bazar/?

[10] R. B. Rusu and S. Cousins "3D/s here: Point Cloud Library (PCL)", Robotics and Automation (ICRA), 2011 IEEE International Conference on, vol. 1, no. 4, (2011) May, pp. 9-13.
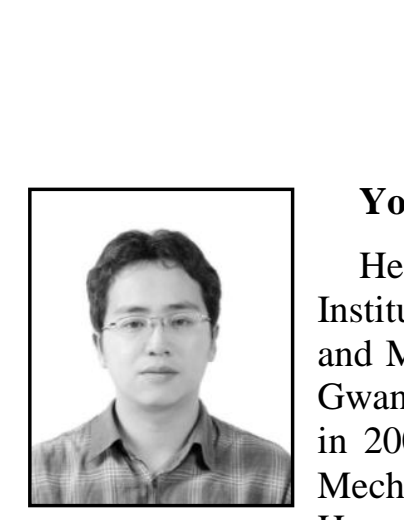

\section{Authors}

\section{Youngho Lee}

He received his BS in Dept. of Mathematics from Korea Advanced Institute of Science and Technology (KAIST), Daejon, Korea, in 1999 and M.S. degree in the Dept. of Informa- tion and communication from Gwangju Institute of Science and Tech- nology (GIST), Gwanju, Korea, in 2001. In 2008, he received his Ph.D. in School of Information and Mechatronics from Gwangju Institute of Science and Technology (GIST). He was a Post-doctoral research fellow and Research Assistant Profrssor at GIST CTI in 2008 and 2009 respectively. Since Sept. 2009, he has been with the Mokpo National University (MNU), where he is a Professor in the Department of Computer Engineering. His research interests include Context-aware computing, HCI, virtual/augmented reality, culture technology, etc. 


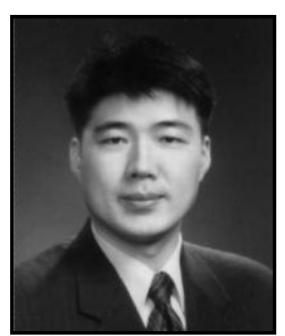

\section{Jongmyong Choi}

He received his M.S. and Ph.D. degree in Computer Science from Soongsil University, Korea, in 1996 and 2003 respec- tively. From 2004 to 2010 he was at Mokpo National University, Ko- rea, as an assistant professor. He is currently an associate professor in Mokpo National University. His research interests are context-aware systems, human computer/robot interaction, social computing, and healthcare.

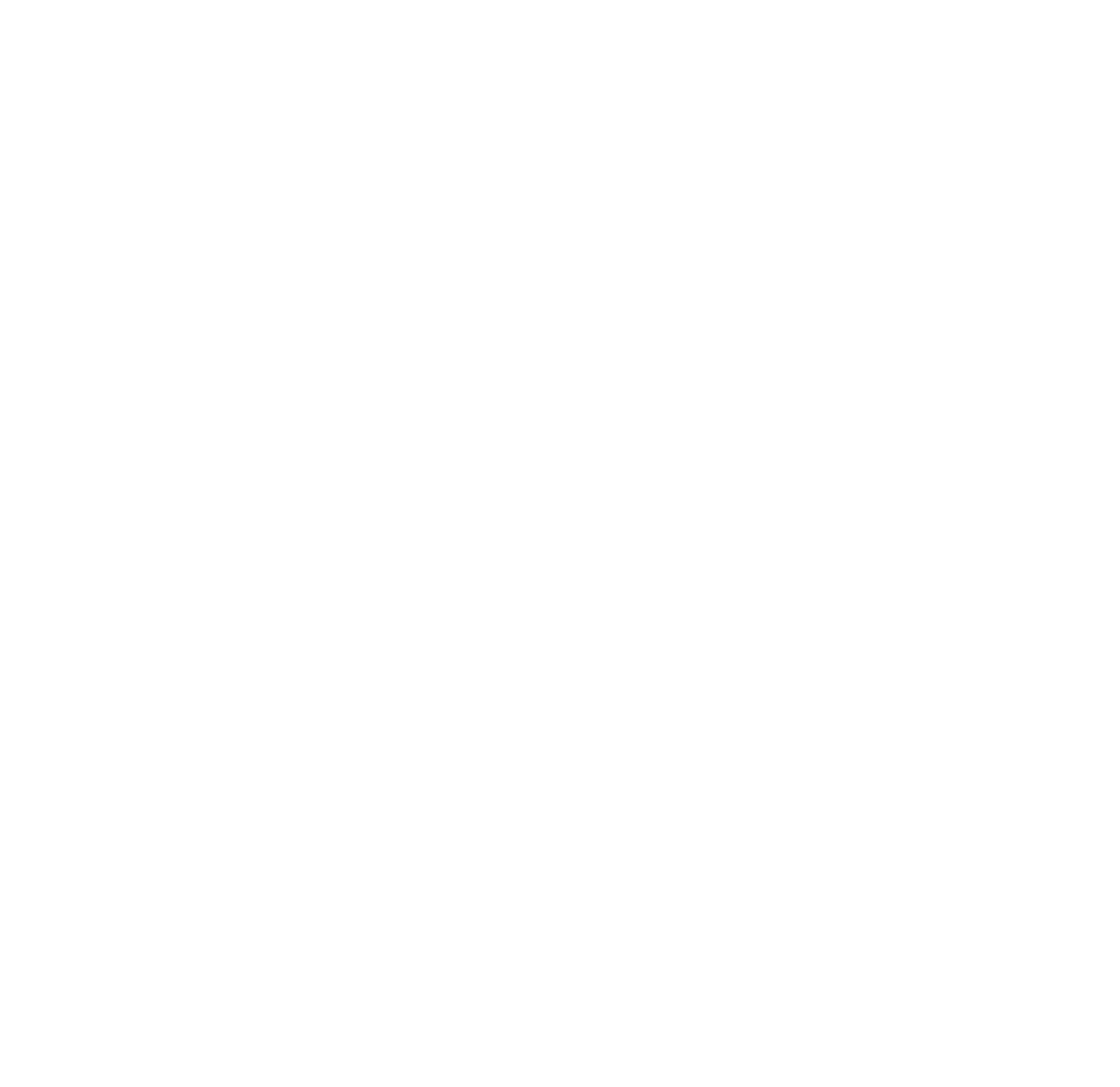

